\title{
LINES ON BRIESKORN-PHAM SURFACES
}

\author{
Guangfeng Jiang, Mutsuo Oka, Duc Tai Pho, and Dirk Siersma
}

\begin{abstract}
By using toric modifications and a result of Gonzalez-Sprinberg and LejeuneJalabert, we answer the following questions completely. On which Brieskorn-Pham surface there exist smooth curves passing through the singular point? If there exist, how "many" and what are the defining equations?
\end{abstract}

\section{Introduction}

Let $(X, 0)$ be an analytic space germ embedded in $\left(C^{n}, 0\right)$, and $(L, 0)$ be a smooth curve germ in $\left(C^{n}, 0\right)$. Since $L$ is locally biholomorphic to a line, we often say that $L$ is a line. If for a singular point $O \in X_{\text {sing, there exists a }}$ line (smooth curve) $L$ in $C^{n}$ such that $O \in L$ and $L \backslash\{O\} \subset X_{\text {reg, we say that } X}$ contains (or has) a line passing through $O$.

On a singular surface $X$ in $C^{3}$ one can not always find a smooth curve passing through (not contained in) the singular locus of $X$. Gonzalez-Sprinberg and Lejeune-Jalabert proved a criterion for the existence of smooth curve on any (two dimensional) surface. We quote this result here for the convenience of the reader. Let $\pi: \tilde{X} \rightarrow(X, 0)$ be the minimal resolution of a singular surface $(X, 0)$. Let $\mathscr{L}$ be the set of lines on $(X, 0)$. For an exceptional divisor $E$, let $\mathscr{L}_{E}$ denote the set of lines on $(X, 0)$ with the strict transform intersecting $E$ transversally. Let $m$ be the maximal ideal of the local ring $\mathcal{O}_{X, 0}$. The cycle $\mathscr{Z}_{X}$ on $\tilde{X}$ defined by the ideal sheaf $m \Theta_{\tilde{X}}$ is called the maximal cycle of $\pi$.

GonZalez-Sprinberg AND LeJeune-JALABert TheOREM $([1,2])$. (1) There exists lines on $(X, 0)$ if and only if the maximal cycle $\mathscr{Z}_{X}$ has at least one reduced component;

(2) The set of all the lines on $(X, 0)$ is a disjoint union of the $\mathscr{L}_{E}$ 's with each $E$ a reduced component in $\mathscr{Z}_{X} . \quad$ And $\mathscr{L}_{E}$ is called the family of lines corresponding to $E$.

1991 Mathematics Subject Classification: 14J17, 32S25, 32S45.

Key words and phrases: line, Brieskorn-Pham surface, toric modification, torsion number.

The first author was supported by JSPS: P98028.

Received January 22, 1999; revised December 10, 1999. 
In $[3,4]$, lines on surfaces with simple singularities are classified by using the classification machinery. All the surfaces with simple or simple elliptic singularities passing through $x$-axis are equivalent to (under the coordinate transformation preserving the $x$-axis) some surfaces defined by explicit equations.

Remark that by the aforementioned theorem many singular surfaces have lines. Among the examples are minimal surfaces, sandwiched surfaces and so on.

In this paper we are interested in the following questions on the so-called Brieskorn-Pham type surfaces, denoted by $G(p, q, r)$, and defined in $C^{3}$ by

$$
x^{p}+y^{q}+z^{r}=0 \text {. }
$$

For what kind of $(p, q, r)$, there are lines on $G(p, q, r)$ ? And for what kind of $(p, q, r)$, there are no lines on $G(p, q, r)$ ? Furthermore, if there are lines, how "many"? What are the defining equations for the lines? We give complete answers to these questions. The method we use here is the toric modifications of surface singularities. We refer the reader to the book [6] for notions and notations.

There is an invariant, called the torsion number [3], attached to each line. This torsion number is defined as follows. For a pair of analytic space germs $(\Sigma, 0) \subset(X, 0)$, embedded in $\left(\boldsymbol{C}^{n}, 0\right)$, defined by the ideals $\mathfrak{g} \supset \mathfrak{h}$ of $\mathcal{O}:=\mathcal{O}_{\boldsymbol{C}^{n}, 0}$ respectively. Denote $\mathcal{O}_{X}:=\mathcal{O} / \mathfrak{h}, \mathcal{O}_{\Sigma}:=\mathcal{O} / \mathfrak{g}$. The image ideal of $\mathfrak{g}$ in $\mathcal{O}_{X}$ is denoted by $\overline{\mathrm{g}}$. The $\mathcal{O}_{\Sigma}$-module $M:=\overline{\mathrm{g}} / \overline{\mathfrak{g}}^{2} \simeq \mathfrak{g} /\left(\mathfrak{g}^{2}+\mathfrak{h}\right)$ is called the conormal module of $\overline{\mathrm{g}}$. If the torsion submodule $T(M)$ of $M$ has finite length, then the length is called the torsion number of $(\Sigma, X)$ and is denoted by $\lambda:=\lambda(\Sigma, X)$.

For two integers $m>n$, let $m / n:=\left[m_{1}, m_{2}, \ldots, m_{k}\right]$ be the continued fraction representation of $m / n$. Define $\Phi(m / n):=\max \left\{i \mid m_{1}=\cdots=m_{i}=2,1 \leq i \leq k\right\}$. Denote the number of families of lines on $G(p, q, r)$ by $\varrho(p, q, r):=\sharp\left\{\mathscr{L}_{E} \neq \emptyset\right\}$. By the above mentioned theorem, $\varrho(p, q, r)=\sharp\left\{\right.$ reduced components in $\left.\mathscr{Z}_{X}\right\}$.

\section{The Newton boundary and the dual Newton diagram}

Let $s:=\operatorname{gcd}(p, q, r)$ and $p_{1}=\operatorname{gcd}(q, r) / s, q_{1}=\operatorname{gcd}(r, p) / s, r_{1}=\operatorname{gcd}(p, q) / s$. Let $\tilde{p}, \tilde{q}, \tilde{r}$ be the integers such that $p / s=\tilde{p} q_{1} r_{1}, q / s=p_{1} \tilde{q} r_{1}, r / s=p_{1} q_{1} \tilde{r}$. Then $\tilde{p}, \tilde{q}, \tilde{r}$ are pairwisely coprime.

The Newton boundary of $G(p, q, r)$ is a triangle with vertices $A(p, 0,0)$, $B(0, q, 0), C(0,0, r)$ on the plane $x / p+y / q+z / r=1$. The dual Newton diagram $\Gamma^{*}$ of $G(p, q, r)$ consists of $E_{1}={ }^{T}(1,0,0), E_{2}={ }^{T}(0,1,0), E_{3}={ }^{T}(0,0,1)$ and $P={ }^{T}\left(p_{1} \tilde{q} \tilde{r}, \tilde{p} q_{1} \tilde{r}, \tilde{p} \tilde{q} r_{1}\right)$, where and in the following we use "T" to indicate that the vector is a column vector.

According to [6, III (6.3)], in order to obtain a canonical toric resolution of the surface $G(p, q, r)$, we need to make a canonical regular simplicial subdivision $\Sigma^{*}$ of $\Gamma^{*}$, which we describe roughly as follows (see [6] and [7] for details). For two vectors $Q_{1}$ and $Q_{2}$ in $Z^{3}$, denote by $\operatorname{det}\left(Q_{1}, Q_{2}\right)$ the greatest common divisor of the absolute values of the $2 \times 2$ minors of the $3 \times 2$ matrix $\left(Q_{1}, Q_{2}\right)$. Obviously, $\operatorname{det}\left(E_{1}, P\right)=\tilde{p}$. If $\tilde{p}>1, E_{1} P$ is not regular and we need to subdivide it. By $[6$, II $(2.1)]$, there exists a unique integer $\tilde{p}>\tilde{p}_{1}>0$ such that the vector $R_{1}:=$ 

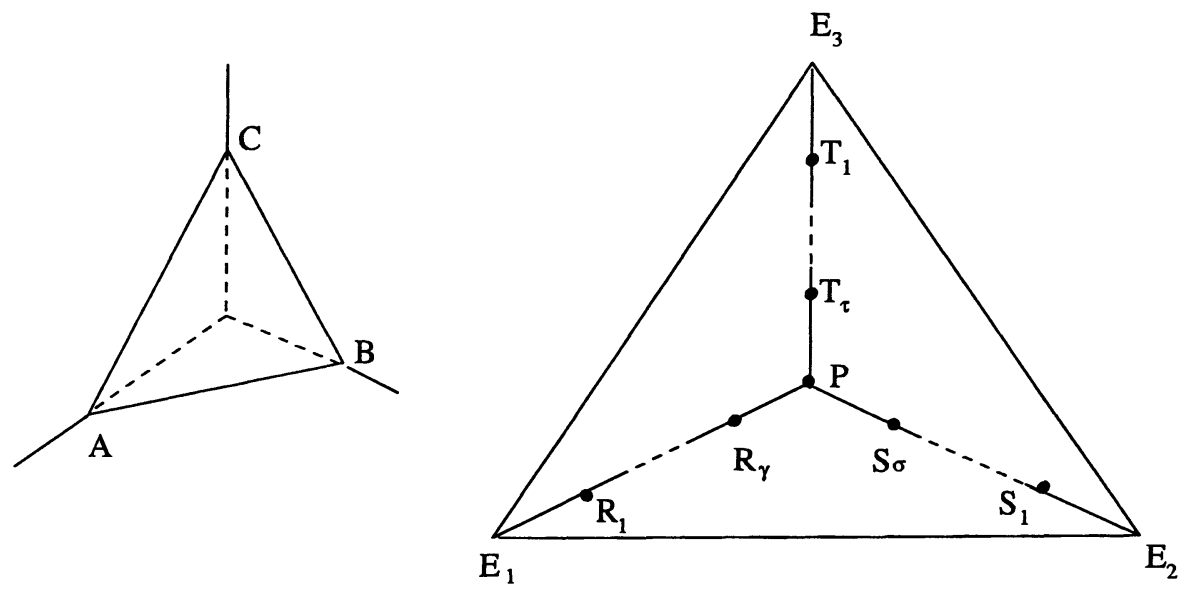

FIGURE 1. Newton boundary and dual Newton diagram of Brieskorn-Pham surface

$\left(P+\tilde{p}_{1} E_{1}\right) / \tilde{p}$ is integral. Then $\operatorname{det}\left(R_{1}, E_{1}\right)=1$ and $\operatorname{det}\left(R_{1}, P\right)=\tilde{p}_{1}<\tilde{p} . \quad$ If $\tilde{p}_{1}>1$, repeat the process for $R_{1} P$, until we obtain a regular subdivision of $E_{1} P$. In this way, we obtain the canonical regular simplicial subdivisions of $E_{1} P, E_{2} P, E_{3} P$. Let $R_{l}={ }^{T}\left(r_{1, l}, r_{2, l}, r_{3, l}\right)(1 \leq i \leq \gamma)$ be the points being added to $E_{1} P$ (see Figure 1). Similarly one adds $S_{j}={ }^{T}\left(s_{1, j}, s_{2, j}, s_{3, J}\right) \quad(1 \leq j \leq \sigma)$ to $E_{2} P$ and $T_{k}=$

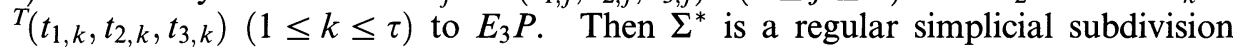
of $\Gamma^{*}$ such that its restriction to $E_{i} P(i=1,2,3)$ is the canonical regular simplicial subdivision of $E_{i} P$ described above.

The dual resolution graph $\mathscr{G}$ of $G(p, q, r)$ is star-shaped with the center $P$, the exceptional divisor determined by $P$. In the following we use the capital letters to denote the points on $\Gamma^{*}$ as well as the exceptional divisors corresponding to them by abusing the symbol. The arms in $\mathscr{G}$ consist of $s p_{1}=\operatorname{gcd}(q, r)$ copies of $P R_{1}, s q_{1}=\operatorname{gcd}(r, p)$ copies of $P S_{1}$ and $s r_{1}=\operatorname{gcd}(p, q)$ copies of $P T_{1}$ (loc. cit.).

LEMMA 1. With the notations as above, we have

$$
r_{l, l} \leq r_{l, l+1} \leq \theta_{l}, \quad s_{l, J} \leq s_{l, J+1} \leq \theta_{l}, \quad t_{l, k} \leq t_{l, k+1} \leq \theta_{l}, \quad l=1,2,3,
$$

where $\theta_{1}:=p_{1} \tilde{q} \tilde{r}, \theta_{2}:=\tilde{p} q_{1} \tilde{r}, \theta_{3}:=\tilde{p} \tilde{q} r_{1}$ are the coordinates of $P$.

Proof. We prove the lemma for the $R_{l}$ 's. Let $\pi$ be the toric modification associated with the above regular simplicial cone subdivision $\Sigma^{*}$. Let $\left(\pi^{*} x\right)=$ $E_{1}+\sum r_{1, i} E\left(R_{l}\right)+p_{1} \tilde{q} \tilde{r} E(P)+D$, where $D$ is the sum of the divisors which do not intersect with $E\left(R_{l}\right)$ 's. Note that the exceptional divisor $E\left(R_{l}\right)$ is a disjoint union of $E\left(R_{l}^{(\alpha)}\right)\left(1 \leq \alpha \leq s p_{1}\right)$. Each $E\left(R_{1}^{(\alpha)}\right)$ possesses the following properties: 1) $E\left(R_{l}^{(\alpha)}\right)$ is isomorphic to $\left.\boldsymbol{P}^{1}, 2\right)$ the self intersection number of $E\left(R_{l}^{(\alpha)}\right)$ is not bigger than -2 by [7] and 3) $E\left(R_{l}^{(\alpha)}\right)$ only intersects with $E\left(R_{l-1}^{(\alpha)}\right)$ and 
$E\left(R_{l+1}^{(\alpha)}\right)$ (here $R_{0}^{(\alpha)}:=E_{1}^{(\alpha)}$, and $R_{\gamma+1}^{(\alpha)}:=P$ ), and $E\left(R_{l}^{(\alpha)}\right) \cdot E\left(R_{l+1}^{(\beta)}\right)=\delta_{\alpha \beta}([6]$ p. 156). Hence from $\left(\pi^{*} x\right) \cdot E\left(R_{1}^{(\alpha)}\right)=1+r_{1,1} E\left(R_{1}^{(\alpha)}\right)^{2}+r_{1,2}=0$, we have $E\left(R_{1}^{(\alpha)}\right)^{2}=$ $-\left(1+r_{1,2}\right) / r_{1,1} \leq-2$ which implies $r_{1,1} \leq r_{1,2}$. From $\left(\pi^{*} y\right) \cdot E\left(R_{1}^{(\alpha)}\right)=0+$ $r_{2,1} E\left(R_{1}^{(\alpha)}\right)^{2}+r_{2,2}=0$, we have $r_{2,2} \geq r_{2,1}$. Similarly, we can prove the other inequalities.

\section{The existence of Lines}

THEOREM 2. Let $s=\operatorname{gcd}(p, q, r) . \quad G(p, q, r)$ has lines if and only if $G(p / s, q / s, r / s)$ has lines.

Proof. It follows from the fact that the two surfaces have the same dual Newton diagram.

TheOREM 3. Assume $\operatorname{gcd}(p, q, r)=1$ and $(\sharp): p \leq q \leq r . \quad G(p, q, r)$ has lines if and only if at least one of the following conditions holds.

I) Two of the three integers $p, q, r$ are coprime and the other one is divisible by at least one of the coprime numbers.

II) The inequality $r>p q / \operatorname{gcd}(p, q)$ holds.

Proof. We continue to use the notations in the beginning of $\S 2$. By the assumption ( $\sharp)$, we have the inequalities $(\sharp \sharp): p_{1} \tilde{q} \tilde{r} \geq \tilde{p} q_{1} \tilde{r} \geq \tilde{p} \tilde{q} r_{1}$.

We first show the necessity.

(a) Assume that there exists 1 in the coordinates of $P$. Then by $(\sharp \sharp)$ we have $\tilde{p} \tilde{q} r_{1}=1$ which implies that $\operatorname{gcd}(p, q)=1$ and $p q \mid r$. This corresponds to a special case of $(\mathrm{I})$.

(b) Let $\operatorname{det}\left(P, E_{1}\right)=\tilde{p}>1$. The coordinates of $R_{1}$ are $r_{1,1}=\left(p_{1} \tilde{q} \tilde{r}+\tilde{p}_{1}\right) / \tilde{p} \geq$ $q_{1} \tilde{r}+\tilde{p}_{1} / \tilde{p}>1$ by $(\sharp \sharp), r_{2,1}=q_{1} \tilde{r} \geq \tilde{q} r_{1}=r_{3,1}$. We assume that $r_{3,1}=1$. This implies that $r_{1}=\tilde{q}=1$. Then we have $p=\tilde{p} q_{1}, q=p_{1}, r=p_{1} \tilde{r}$, from which we have $\operatorname{gcd}(p, q)=1$ and $q \mid r$. This corresponds to a case of (I).

(c) In case $\operatorname{det}\left(P, E_{2}\right)=\tilde{q}>1$, the coordinates of $S_{1}$ are $s_{2,1}=\left(\tilde{p} q_{1} \tilde{r}+\tilde{q}_{1}\right) / \tilde{q}$ $>1, s_{1,1}=p_{1} \tilde{r} \geq s_{3,1}=\tilde{p} r_{1}$. Assume that $s_{3,1}=\tilde{p} r_{1}=1$. Then $p \mid r$ and $\operatorname{gcd}(p, q)=$ 1. This is also a case of $(\mathrm{I})$.

(d) If $\operatorname{det}\left(P, E_{3}\right)=\tilde{r}>1$, the coordinates of $T_{1}$ are $t_{1,1}=p_{1} \tilde{q} \geq t_{2,1}=\tilde{p} q_{1}$ and $t_{3,1}=\left(\tilde{p} \tilde{q} r_{1}+\tilde{r}_{1}\right) / \tilde{r}$. In this case, we have only two possibilities. (d-1) Firstly, assume that $t_{2,1}=1$ which is equivalent to $p \mid q$ and $\operatorname{gcd}(p, r)=1$. This is a case of (I). Since $\operatorname{gcd}(p, q, r)=1$, this is also a case of (II) if $p>1$. (d-2) Secondly, assume that $t_{3,1}=1$. This implies $\tilde{r}=\tilde{p} \tilde{q} r_{1}+\tilde{r}_{1}$ for some $\tilde{r}>\tilde{r}_{1}>0$, which implies that $\tilde{r}>\tilde{p} \tilde{q} r_{1}$. This implies that $r>p q / \operatorname{gcd}(p, q)$, which corresponds to (II).

Note that all the coordinates of $R_{l}\left(i>1\right.$ in case $\left.\tilde{p}_{1}>1\right)$ and that of $S_{j}$ $\left(j>1\right.$ in case $\left.\tilde{q}_{1}>1\right)$ are bigger than one. Also the first two coordinates of $T_{k}$ $\left(k>1\right.$ in case $\left.\tilde{r}_{1}>1\right)$ are bigger than one. By lemma $1, t_{3, k}=1$ implies that $t_{3, \alpha}=1$ for all $\alpha=1, \ldots, k-1$. Hence $r>p q / \operatorname{gcd}(p, q)$. 
We show the sufficiency. Assume first that (II): $r>p q / \operatorname{gcd}(p, q)$. Then $\tilde{r}>\tilde{p} \tilde{q} r_{1}$ and $\tilde{r}_{1}=\tilde{r}-\tilde{p} \tilde{q} r_{1}$ and we get $t_{3,1}=1$. Next, assume that (I) is satisfied. Then by $(\sharp)$, either $(1) \operatorname{gcd}(p, q)=1$ and $p \mid r$ or $q \mid r$ or $(2) \operatorname{gcd}(p, r)=1$ and $p \mid q$. (1) corresponds to a case of (a), (b), or (c) and (2) corresponds to a case of (a) or (d). For example assume that $\operatorname{gcd}(p, q)=1$ and $p \mid r$. This implies that $r_{1}=1$ and $\tilde{p} q_{1} \mid \tilde{r} p_{1} q_{1}$. Thus $\tilde{p}=1$ and this corresponds to (c) (respectively to (a)) if $\tilde{q}>1$ (resp. if $\tilde{q}=1$ ). The other cases are treated similarly.

Remark 4. The case that $p=1$ is a special case of (I) and (II).

\section{Equations, numbers and torsion numbers of the lines}

We continue to use the notations in the beginning of $\S 2$ and to assume $p \leq q \leq r$. Gonzalez-Sprinberg and Lejeune-Jalabert theorem also gives a way to find the defining equations of the lines (as curves in $C^{3}$ ) on a surface. The computation of the torsion number can be done as follows. For a line $L$ with parameterization $x=x(t), y=y(t), z=z(t)$, one may assume that $d x / d t(0) \neq$ 0 . By the inverse function theorem, we have $t=t(x)$. Hence the line $L$ can be defined by $y-\phi(x)=0$ and $z-\psi(x)=0$. We choose the following local coordinate transformation of $\left(C^{3}, 0\right): x^{\prime}=x, y^{\prime}=y-\phi(x), z^{\prime}=z-\psi(x)$. The defining ideal of $L$ is thus $\mathfrak{g}=\left(y^{\prime}, z^{\prime}\right)$. The defining equation of the surface containing $L$ can be written as $h \equiv a x^{\prime} y^{\prime}+b x^{\prime} z^{\prime} \bmod g^{2}$. Then $\lambda=$ $\operatorname{dim}_{C} \mathcal{O} /\left(x^{\prime}(a, b)+\mathfrak{g}\right)($ see $[4])$.

Lemma 5. $\varrho(p, p, p)=1$ and the line can be parameterized by

$$
x=b t, \quad y=c t, \quad z=t, \quad \text { with } b^{p}+c^{p}+1=0,
$$

and the torsion number $\lambda=p-1$.

Proof. The proof is trivial and is omitted.

LeMma 6. 1) If $p>1$, then $\varrho(p, p q, p q r)=p(r-1)+1$. Let $T_{0}:=E_{3}, T_{r}$ $:=P$, and $T_{1}, \ldots, T_{r-1}$ be the points on $E_{3} P$ to make the canonical regular simplicial subdivision of $E_{3} P$. Then to each $T_{k}$ correspond $p$ reduced components $T_{k}^{(i)}(r>k>0)$ in the maximal cycle of $G(p, p q, p q r)$. The torsion numbers for the lines in $\mathscr{L}_{T_{k}^{(i)}}(k$ fixed $)$ are the same: $\lambda=k(p-1) q$;

2) If $p>1$ and $q \nmid r$, then $\varrho(p, p q, p r)=p(1+[r / q])$. Let $T_{0}:=E_{3}$, and $T_{1}, \ldots, T_{[r / q]}$ be the points on $E_{3} P$ to make the canonical regular simplicial subdivision of $E_{3} P$. Then to each $T_{k}={ }^{T}(k q, k, 1)$ correspond $p$ reduced components $T_{k}^{(i)}(k>0)$ in the maximal cycle of $G(p, p q, p r)$. The torsion numbers of the lines in $\mathscr{L}_{T_{k}^{(i)}}$ for each fixed $k(k=1, \ldots,[r / q])$ are the same $\lambda=k(p-1) q$.

Let $S_{1}$ be on $P E_{2}$ (see Figure 1) with 1 as the last coordinate. There are $p$ reduced components $S_{1}^{(i)}(i=1, \ldots, p)$ in the maximal cycle corresponding to $S_{1}$. 
TABLE 1. Number of lines on Brieskorn-Pham surfaces

\begin{tabular}{|c|c|c|}
\hline \multicolumn{2}{|r|}{ Conditions } & $\varrho(p, q, r)$ \\
\hline \multirow{4}{*}{$\begin{array}{l}\operatorname{gcd}(p, q)=1 \\
1<p<q \leq r\end{array}$} & $r=q$ & $q$ \\
\hline & $r>q, p q \mid r$ & $\frac{r}{p q}=1+\Phi\left(\frac{r}{r-p q}\right)$ \\
\hline & $r>q, p \nmid r, q \mid r$ & {$\left[\frac{r}{p q}\right]+q=1+\Phi\left(\frac{r}{r-p q}\right)+q$} \\
\hline & $r>q, p \mid r, q \nmid r$ & {$\left[\frac{r}{p q}\right]+p=1+\Phi\left(\frac{r}{r-p q}\right)+p$} \\
\hline $\begin{array}{l}r>\frac{p q}{\operatorname{gcd}(p, q)} \\
1<p \leq q \leq r \\
\operatorname{gcd}(p, q, r)=1\end{array}$ & $\begin{array}{l}\operatorname{gcd}(p, q)=1, p \nmid r, q \nmid r \\
\text { or } \\
\operatorname{gcd}(p, q)>1\end{array}$ & $\begin{array}{l}\operatorname{gcd}(p, q)\left[\frac{r \operatorname{gcd}(p, q)}{p q}\right] \\
=\operatorname{gcd}(p, q)\left\{1+\Phi\left(\frac{r}{r-p q / \operatorname{gcd}(p, q)}\right)\right\}\end{array}$ \\
\hline
\end{tabular}

The torsion numbers of the lines in $\mathscr{L}_{S_{1}^{(i)}}$ are the same: $\lambda=\min \{(p-1) r$, $(p q-1)(1+[r / q])\}$.

Proof. We leave the easy proof to the reader.

In the following, we assume that $\operatorname{gcd}(p, q, r)=1$ and $1<p \leq q \leq r$. The exact numbers of families of lines are given in Table 1. The proof is in lemma 7-11.

LeMmA 7. Let $1<p<q=r$ and $\operatorname{gcd}(p, q)=1$. There are $q$ reduced components $R_{1}^{(i)}$ in the maximal cycle corresponding to $R_{1}$ (see figure 1$)$. And the $q$ families of lines on $G(p, q, q)$ can be parameterized by

$$
x=c t^{1+[q / p]}, \quad y=t, \quad z=\omega_{\beta}\left(1+c^{p} t^{p-\delta}\right)^{1 / q} t, \quad \beta=1, \ldots, q,
$$

where $c \neq 0$ is a constant, $\omega_{\beta}$ is a root of $\omega^{q}=-1$, and $\delta=q-p[q / p]$. The torsion numbers of these lines are the same $\lambda=\min \{q-1,(p-1)(1+[q / p])\}$.

Proof. We omit the easy proof.

LeMma 8. Let $\operatorname{gcd}(p, q)=1$ and $p q \mid r$. The maximal cycle is reduced and consists of $T_{k+1}={ }^{T}((k+1) q,(k+1) p, 1) \quad(k=0, \ldots, \tilde{r}-1=r / p q-1) . \quad$ The lines in $\mathscr{L}_{T_{k+1}}$ can be parameterized by

$$
x=c^{k q} u^{(1+\alpha q+k p q) / p} t^{(k+1) q}, \quad y=c^{k p} u^{\alpha+k p} t^{(k+1) p}, \quad z=c u t,
$$

where $0 \leq \alpha<p$ such that $(1+\alpha q+k p q) / p$ is an integer, $c \neq 0$ is a constant and 
$u=u(t)$ is a unit satisfying $1+u+u^{r-(\alpha+p k) q} v^{r-(k+1) p q} w^{r-k p q}=0 . \quad$ The torsion number for the lines in $\mathscr{L}_{T_{k+1}}$ is $\lambda=(k+1)(p-1) q$.

Proof. In this case $P={ }^{T}(\tilde{r} q, \tilde{r} p, 1)$ and $\operatorname{det}\left(P, E_{1}\right)=\operatorname{det}\left(P, E_{2}\right)=1$, $\operatorname{det}\left(P, E_{3}\right)=\tilde{r}$. Let $0 \leq \alpha, \beta<p$ such that

$$
M_{k}=\frac{1}{p}\left(E_{1}+\alpha T_{k+1}+\beta T_{k}\right)={ }^{T}\left(\frac{1+\alpha q+k p q}{p}, \alpha+p k, 1\right)
$$

is integral. From the chart determined by $M_{k}, T_{k+1}, T_{k}$, one can do all the computations.

Lemma 9. Let $\operatorname{gcd}(p, q)=1$ and $p \nmid r, q \mid r$. In the maximal cycle there are $q$ reduced components $R_{1}^{(i)}(1 \leq i \leq q)$ corresponding to $R_{1}={ }^{T}((r+\delta) / p, r / q, 1)$, where $0<\delta<p$ such that $(r+\delta) / p$ is an integer. The lines in $\mathscr{L}_{R_{1}^{(i)}}$ can be parameterized as

$$
x=c t^{(r+\delta) / p}, \quad y=\omega_{i}\left(1+c^{q} t^{\delta}\right)^{1 / q} t^{r / q}, \quad z=t,
$$

where $c \neq 0$ is a constant and $\omega_{i}$ is a root of $\omega^{q}=-1, i=1, \ldots, q$. The torsion numbers are the same: $\lambda=\min \{(p-1)([r / q]+1),(q-1) r / q\}$. Moreover, if $r>$ $p q$, in the maximal cycle there are also reduced components corresponding to $T_{k+1}$ $={ }^{T}((k+1) q,(k+1) p, 1) \quad(k=0, \ldots,[r /(p q)]-1)$. The parameterizations and the torsion numbers of the lines in $\mathscr{L}_{T_{k+1}}$ are the same as in lemma 8.

Proof. One only needs to prove the statement about $\mathscr{L}_{R_{1}^{(1)}}$, which can be done from the chart determined by $E_{1}, E_{2}, R_{1}$.

LEMma 10. Let $\operatorname{gcd}(p, q)=1$ and $p \mid r, q \nmid r$. In the maximal cycle, there are $p$ reduced components $S_{1}^{(j)}(1 \leq j \leq p)$ corresponding to $S_{1}={ }^{T}(r / p,(r+\delta) / q, 1)$, where $0<\delta<q$ such that $S_{1}$ is integral. The lines in $\mathscr{L}_{S_{1}^{(J)}}$ can be parameterized by

$$
x=\omega_{j}\left(1+c^{q} t^{\delta}\right)^{1 / p} t^{r / p}, \quad y=c t^{(r+\delta) / q}, \quad z=t,
$$

where $c \neq 0$ is a constant and $\omega_{j}$ is a root of $\omega^{p}=-1, j=1, \ldots, p$. The torsion numbers are the same: $\lambda=\min \{(q-1)([r / q]+1),(p-1) r / p\}$. Moreover, if $r>$ $p q$, there are also $[r /(p q)]$ reduced components in the maximal cycle corresponding to $T_{k+1}={ }^{T}((k+1) q,(k+1) p, 1)(k=0, \ldots,[r /(p q)]-1)$. The parameterizations and the torsion numbers of the lines in $\mathscr{L}_{T_{k+1}}$ are the same as in lemma 8.

Proof. Similar to that of lemma 9.

LeMma 11. Let $\operatorname{gcd}(p, q, r)=1, \quad 1<p \leq q \leq r$ and $r>p q / \operatorname{gcd}(p, q)$. Assume that either $\operatorname{gcd}(p, q)=1, p \nmid r, q \nmid r$ or $\operatorname{gcd}(p, q)>1$. Then to each

$$
T_{k+1}=\left(\frac{(k+1) q}{\operatorname{gcd}(p, q)}, \frac{(k+1) p}{\operatorname{gcd}(p, q)}, 1\right), \quad\left(k=0, \ldots,\left[\frac{r \operatorname{gcd}(p, q)}{p q}\right]-1\right)
$$


correspond $\operatorname{gcd}(p, q)$ reduced components $T_{k+1}^{(\gamma)}(\gamma=1, \ldots, \operatorname{gcd}(p, q))$ in the maximal cycle. And they are the only reduced components in the maximal cycle. For fixed $k$, the lines in $\mathscr{L}_{T_{k+1}^{(i)}}$ can be parameterized by

$$
x=c^{k \bar{q}} u_{\gamma}^{(1+(k \bar{p}+\alpha) \bar{q}) / \bar{p}} t^{(k+1) \bar{q}}, \quad y=c^{k \bar{p}} u_{\gamma}^{k \bar{p}+\alpha} t^{(k+1) \bar{p}}, \quad z=c u_{\gamma} t, \quad \gamma=1, \ldots, r_{1},
$$

where $u_{\gamma}$ is a unit satisfying $1+u^{r_{1}}+c^{r-k \bar{p} q} u^{r-(k \bar{p}+\alpha) q} t^{r-(k+1) \bar{p} q}=0$, and $r_{1}=$ $\operatorname{gcd}(p, q), \bar{p}=p / r_{1}, \bar{q}=q / r_{1}, 0 \leq \alpha<\bar{p}$ such that $(1+(k \bar{p}+\alpha) \bar{q}) / \bar{p}$ us an integer. And the torsion numbers of the lines in $\mathscr{L}_{T_{k+1}^{(\gamma)}}$ are: $\lambda=(k+1)(p-1) q / \operatorname{gcd}(p, q)$.

Proof. Notice that all the coordinates of $R_{l}$ and $S_{j}$ are greater than 1 and $T_{k+1}$ is as in the statement of the lemma $(k=0, \ldots,[(r \operatorname{gcd}(p, q)) /(p q)]-1)$. Denote $T_{0}:=E_{3}$. Let $0 \leq \alpha, \beta<\bar{p}$ be the integers such that

$$
M_{k}:=\frac{1}{\bar{p}}\left(E_{1}+\alpha T_{k+1}+\beta T_{k}\right)={ }^{T}\left(\frac{1+(k \bar{p}+\alpha) \bar{q}}{\bar{p}}, k \bar{p}+\alpha, 1\right)
$$

is integral. Then the simplex determined by $M_{k}, T_{k+1}, T_{k}$ is regular. From this chart, one can obtain the given parameterization of the lines in $\mathscr{L}_{T_{k+1}^{(j)}}$ by a calculation.

COROLlaRY 12. If any two of the $p, q, r$ are coprime and $q=p+\alpha$, $r=q+\beta$ with $0<\alpha<p, 0<\beta<q$, there do not exist smooth curves on $G(p, q, r)$.

Proof. We give an elementary proof. Let $C$ be a smooth curve in $\left(C^{3}, 0\right)$, parameterized by

$$
x=x(t)=\sum_{k=1}^{\infty} a_{k} t^{k}, \quad y=y(t)=\sum_{k=1}^{\infty} b_{k} t^{k}, \quad z=z(t)=\sum_{k=1}^{\infty} c_{k} t^{k}, \quad|t|<\varepsilon .
$$

Then $C \subset G(p, q, r)$ if and only if

$$
[x(t)]^{p}+[y(t)]^{q}+[z(t)]^{r}=0 \text { for all }|t|<\varepsilon .
$$

From this and the assumption on $p, q, r$, one can deduce that $a_{1}=b_{1}=$ $c_{1}=0$.

Example 13. 1) On $E_{8}$ surface $G(2,3,5)$ there do not exist lines.

2) On the surfaces $G(2,3,7)$, there exists one family of lines. A regular subdivision of the dual Newton diagram is given in Figure 2. Where $P=$ $T(21,14,6), \quad Q={ }^{T}(11,7,3), \quad R=T_{(7,5,2), \quad S=T}(3,2,1), \quad S_{1}={ }^{T}(1,1,1), \quad S_{2}=$ ${ }^{T}(2,2,1), \quad S_{3}={ }^{T}(2,1,2), \quad S_{4}={ }^{T}(2,1,1), \quad T={ }^{T}(4,3,1) . \quad$ Only the exceptional divisors corresponding to $P, Q, R, S$ have non-empty intersections with the strict transform of the surface $G(2,3,7)$. On the chart corresponding to the triangle $\sigma=\Delta Q P S$, we have the modification map represented by

$$
x=u^{11} v^{21} w^{3}, \quad y=u^{7} v^{14} w^{2}, \quad z=u^{3} v^{6} w
$$

where $(u, v, w)$ is the coordinates on the chart corresponding to $\sigma$. The strict 


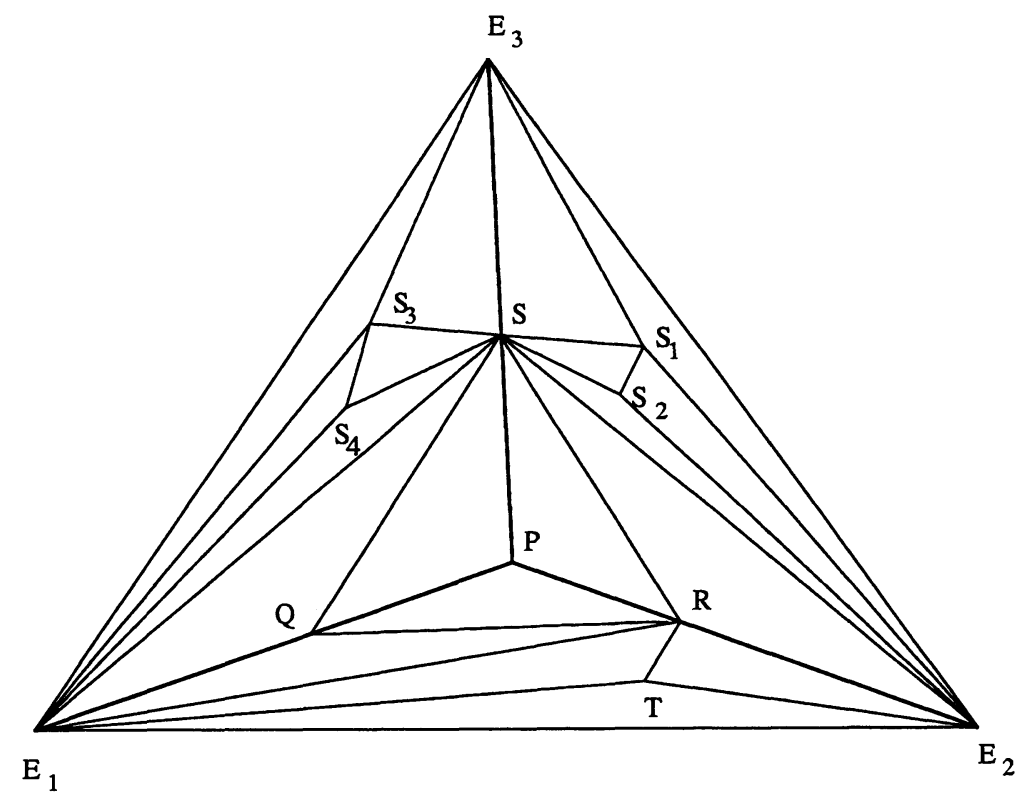

FIGURE 2

transform of $G(2,3,7)$ is defined by $u+w+1=0$. The exceptional divisor corresponding to $S$ is defined by $u+1=0, w=0$. The blowing down of the line $v=c \neq 0, u+w+1=0$ is the smooth curve in $\left(\boldsymbol{C}^{3}, 0\right)$ :

$$
x=-c^{21}(1+t)^{11} t^{3}, \quad y=-c^{14}(1+t)^{7} t^{2}, \quad z=-c^{6}(1+t)^{3} t,
$$

where $t \in C$ is the parameter. This curve is on $G(2,3,7)$ with torsion number 3.

Remark 14. If one applies the formula in lemma 11 to this example, one gets a different parameterization. This shows that the parameterizations of the lines in the same family might be different. This is because the toric modifications of the surface and the chart used might be different. However, the torsion numbers are invariant.

\section{REFERENCES}

[1] G. GonZalez-Sprinberg and M. Lejeune-Jalabert, Courbes lisses sur les singularités de surface, C. R. Acad. Sc1. Parı, t. Sétie, I, 318 (1994) 653-656.

[2] G. GonZalez-SPRINBERG AND M. LeJEunE-JALABERT, Families of smooth curves on surface singularities and wedges, Ann. Polonici Math., 67 (1997) 179-190.

[ 3 ] G. JiANG, Functions with non-1solated singularities on singular spaces, Thesis, Universiteit Utrecht, 1998. 
[ 4 ] G. Jiang ANd D. Siersma, Local embeddings of lines in singular hypersurfaces, Ann. Inst. Fourier (Grenoble), 49 (1999) 1129-1147

[5] H. B. Laufer, Normal Two-dimensional Singularities, Ann. Math. Stud., 71, Princeton Unıv. Press, Princeton, 1971.

[6] M. OKA, Non-degenerate Complete Intersection Singularity, Hermann, Parıs, 1997

[7] M. OKA, On the resolution of the hypersurface singularities, T. Suwa and P, Wagreich eds., Complex Analytıc Singularitıes, Adv. Stud. Pure Math., 8, Kinokuniya, Tokyo, 1987, p. 405436.

Jiang: Department of Mathematics TOKYo METROPOLITAN UNIVERSITY Minami-OHSAWA, HaChioji, TOKYO 192-0397, JAPAN e-mail: jiang@comp.metro-u.ac.jp
(Current Adress)

Department of Mathematics and Physics FACUlTy OF SCIENCE Beijing University of Chemical Technology BeIJING, 100029

P R. CHINA

e-mail: jianggf@gate930.buct.edu.cn

Oka: Department of Mathematics TOKyo Metropolitan UnivERSITy Minami-OHSAWA, HaChioJi, TOKYO 192-0397, JAPAN e-mail: oka@comp.metro-u.ac.jp

Duc: Department of Mathematics Tokyo Metropolitan University Minami-OHSAWA, HaChioJi, TOKYO 192-0397, JAPAN e-mail: pdta1@comp.metro-u.ac.jp

Siersma: DePartment of Mathematics UTRECHT UNIVERSITY BudaPestlaAN 6, P.O. Box 80.010, 3508 TA Utrecht, The Netherlands e-mail: siersma@math.uu.nl 